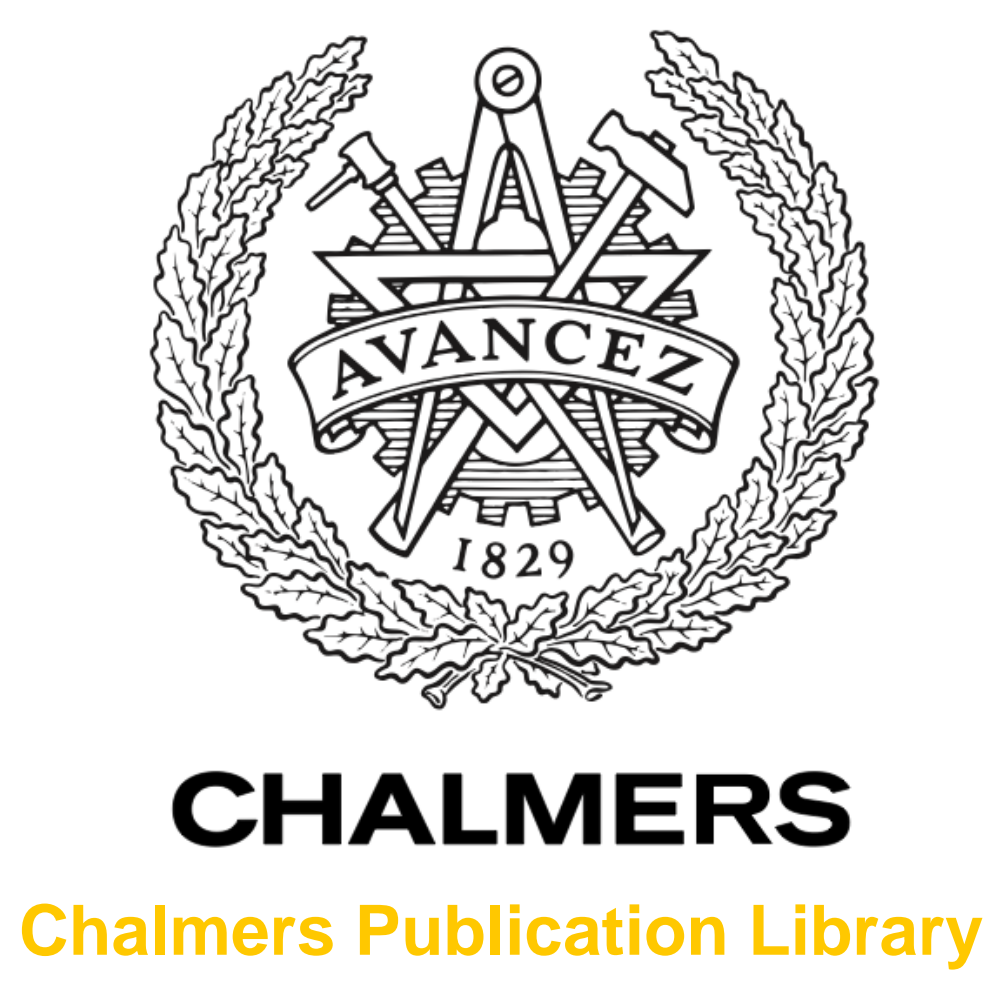

\title{
Enhanced accuracy in dielectric response material characterization by air reference method
}

This document has been downloaded from Chalmers Publication Library (CPL). It is the author's version of a work that was accepted for publication in:

IEEE transactions on dielectrics and electrical insulation (ISSN: 1070-9878)

Citation for the published paper:

Xu, X. ; Bengtsson, T. ; Blennow, J. (2013) "Enhanced accuracy in dielectric response material characterization by air reference method". IEEE transactions on dielectrics and electrical insulation, vol. 20(3), pp. 913-321.

http://dx.doi.org/10.1109/tdei.2013.6518960

Downloaded from: http://publications.lib.chalmers.se/publication/203091

Notice: Changes introduced as a result of publishing processes such as copy-editing and formatting may not be reflected in this document. For a definitive version of this work, please refer to the published source. Please note that access to the published version might require a subscription.

Chalmers Publication Library (CPL) offers the possibility of retrieving research publications produced at Chalmers University of Technology. It covers all types of publications: articles, dissertations, licentiate theses, masters theses, conference papers, reports etc. Since 2006 it is the official tool for Chalmers official publication statistics. To ensure that Chalmers research results are disseminated as widely as possible, an Open Access Policy has been adopted.

The CPL service is administrated and maintained by Chalmers Library. 


\title{
Enhanced Accuracy in Dielectric Response Material Characterization by Air Reference Method
}

\author{
Xiangdong Xu, Tord Bengtsson', Jörgen Blennow and Stanislaw M. Gubanski \\ Department of Materials and Manufacturing Technology \\ Chalmers University of Technology, SE 41296 Gothenburg, Sweden \\ ${ }^{1}$ also with ABB Corporate Research, SE 72178 Västerås, Sweden
}

\begin{abstract}
Dielectric response measurements are an important technique to characterize dielectric materials. However, the electrode arrangements as well as the accuracy of the measurement setup limit the precision of this characterization, especially in case of solid dielectric materials. An air reference method and a contact-free electrode arrangement are described in this paper to enhance the dielectric characterization accuracy by avoiding problems introduced by electrode contacts. It is shown that by performing a calibration with electrode gap filled with air under the same conditions as the material is tested, the air reference method can improve the measurement accuracy substantially. This type of approach also eliminates the need of a detailed model of the analog measurement circuit. In conjunction with the contact-free measurements, the approach allows for avoiding complicated and time-consuming sample preparation procedures. The measurement methodology as well as the electrode arrangement and error increase estimates are presented and evaluated by using different dielectric response instruments and materials.
\end{abstract}

Index Terms - Dielectric material characterization, dielectric frequency response, air reference method, shunt characterization, contact-free electrode arrangement.

\section{INTRODUCTION}

DIELECTRIC response measurements are a significant analysis technique for dielectric materials studies. These measurements can be performed both in time and frequency domain. In the frequency domain, these are also known as frequency domain spectroscopy (FDS) [1,2] and allow for extracting the complex impedance of tested objects under excitation by voltage signals with variable frequency. By measuring excitation voltage and resulting current, the complex impedance can be calculated over the used frequency spectrum.

One main area of FDS application is the selection and development of dielectric materials appropriate for various constructions, where the relative permittivity $\left(\varepsilon_{\mathrm{r}}\right)$ and loss factor $(\tan \delta)$ of the material are of interest. These can be obtained from FDS measurement designed to characterize dielectric materials and are important design parameters in many electrical insulation applications [3-5]. The loss factor of modern insulation materials is often in the $10^{-4}$ range, which imposes severe demands on the measurement instrumentation. Indeed, many commercial FDS instruments have a resolution

Manuscript received on 22 March 2012, in final form 23 January 2013. that is at or above this range. Moreover, due to the possible appearance of surface contact problem between material and electrodes, the electrode arrangements for precision characterization of a dielectric material become complicated and time consuming.

In this paper, an air reference procedure is demonstrated that enables contact-free, fast dielectric characterization of a piece of dielectric material in the frequency domain with enhanced accuracy by eliminating the need of a detailed model of the analog measurement circuit.

Though contact-free electrode arrangement has been discussed almost over a century [6,7], the significant error increase due to presence of an air layer is probably the main reason why it is not in common use. With the help of highly accurate signal measurement techniques and an air reference method, contact-free electrode arrangement for dielectric material characterization can however be practically applied with sufficient accuracy. The loss of precision due to the introduction of an air gap is discussed in this paper in some details.

\section{DIELECTRIC CHARACTERIZATION OF MATERIALS}

The principle of dielectric response measurements is based on a voltage-current measurement, from which the complex impedance frequency response of the test object is obtained 


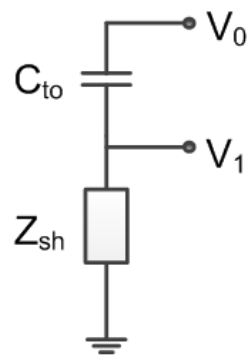

Figure 1. Principle circuit for FDS measurement of a test object $\left(\mathrm{C}_{\mathrm{to}}\right)$. The shunt impedance $\left(Z_{\mathrm{sh}}\right)$ is used to measure the current passing through the test object.

by sweeping in the desired frequency range. The basic circuit for dielectric response measurements is shown in Figure 1.

By applying a voltage with a specified frequency $V_{0}(\omega)$ and measuring the voltage waveform of $V_{0}(\omega)$ and $V_{l}(\omega)$, the complex impedance of the test object $C_{t o}(\omega)$ is calculated through equation (2.1).

$$
C_{t o}^{*}=\frac{V_{1}}{j \omega\left(V_{0}-V_{1}\right) Z_{s h}}
$$

Though dielectric frequency response is an important technique, some factors in the customary implementation are limiting its value. For example, a well-defined voltage source is needed to generate a current response at various frequencies, which makes on-line monitoring impossible; sweeping of a frequency spectrum is time consuming and requires a well-controlled testing environment. In addition to those limitations, modeling of the whole analog measurement circuit and material contact are two hurdles in high precision FDS dielectric materials characterization.

An air reference method provides a solution which enables contact-free dielectric response measurements using a calibration with air as a tested material to eliminate modeling of the analog circuit.

\subsection{CIRCUIT CHARACTERIZATION}

Shunt modeling is a bottleneck in high precision dielectric response measurement as analog circuits are practically impossible to characterize with accuracy substantially below the percent range. Further, shunt impedance variation due to its heating by current flowing through it is another cause of reduced precision. An example on how the shunt used in this work behaves in such situations is illustrated in Figure 2. One may clearly see that at least $1000 \mathrm{~s}$ are needed for the impedance to stabilize. During this time, the change of the shunt impedance is about 5 times the noise level. Here, it is clearly favorable to perform a calibration when the instrument has stabilized under the correct current load and other ambient conditions, which is made possible by the air reference method.

Active operational amplifiers are presently widely used for the current measurement as these provide much lower input impedance and thus reduce the voltage drop over a high impedance shunt. However, the temperature dependence of the electrical circuit and the need to accurately characterize the measurement circuit are not eliminated by this technique.

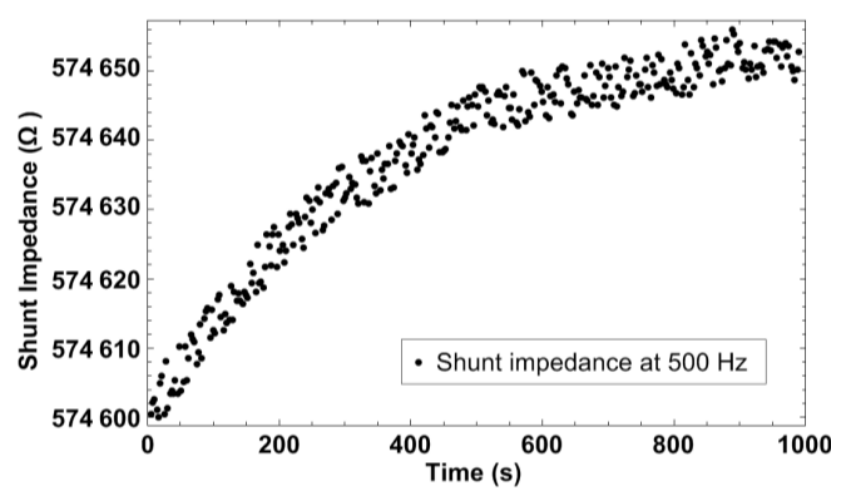

Figure 2. The measured impedance of a current shunt at $500 \mathrm{~Hz}$ during $1000 \mathrm{~S}$ of the same current load. The noise level is visible by the distribution of individual measurements.

\subsection{ELECTRODE CONTACTS}

Material characterization is often hampered by the intricate electrode arrangements required for reliable measurements as the used electrodes should be in proper contact to the test object for avoiding artifacts. If the tested sample is a "soft" material, the proper material contact with electrodes may not be an issue. However, the applied pressure from the electrodes may deform the sample and possibly change the measured permittivity. If, on the other hand, the tested sample is a "stiff" material, deformation of the sample under pressure may not be significant. However, as illustrated in Figure 3, areas with no contact between electrodes and sample surface will create current paths along the sample surface that yield a higher measured loss factor due to the appearance of a series connected resistance at the surface.

Many different electrode arrangements have been described and used for achieving a proper material contact [7]. Among them "liquid" electrodes, i.e., conducting material on the surface, such as silver painting or sputtering [8], conductive glue with copper tape [9], water with $\mathrm{NaCl}$ [10] are popular solutions. A liquid metal electrode may however change the material properties and be toxic [7], for instance, thin samples may absorb a paint solvent and vapors of mercury are toxic. Comb electrodes [11] can provide higher test capacitance and proper surface contact, but they are hard to fabricate and not easy to recycle. The electrodes mentioned above share one common limitation: their preparation is time consuming. Some other electrode systems, such as for example semiconducting rubber electrodes [12], may also deform the sample when pressed against it and will yield increased loss factor at high frequencies due to the electrode material resistance.

A simple solution to the contact problem is to entirely avoid

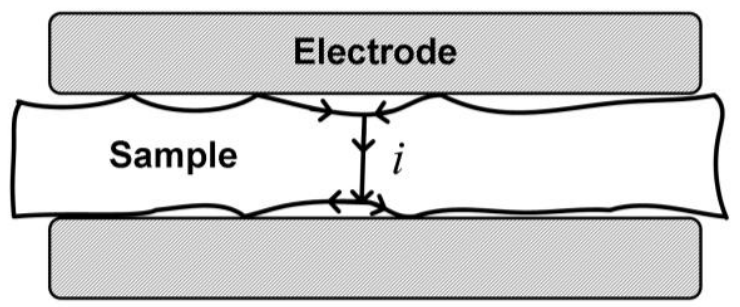

Figure 3. Surface current paths due to insufficient contact between sample and electrodes. 
any direct contact between the material and the electrodes, thus measuring the combined response of a small air gap and the sample. Without any pressure applied on the tested sample and by elimination of the surface conductivity, a contact-free electrode arrangement seems like the ideal solution for avoidance of the material contact problem in dielectric response measurements. A complication is however that the result must be compensated for the presence of air gap to obtain the material permittivity and this compensation is discussed below.

Further, the introduction of an air gap in the electrode system will decrease the useful response signal. Thus, a higher precision instrument is required so that the remaining precision is sufficient to determine the loss factors of interest.

\subsection{CONTACT-FREE MEASUREMENT}

The idea of a contact-free dielectric response measurement is to place a piece of a flat dielectric material on the bottom electrode without direct contact with the top electrode, as shown in Figure 4. As there is no pressure applied from top electrode, deformation of the sample can be neglected. Moreover, without any direct surface contact between sample and electrode, influence of the surface conductivity is limited.

A partially filled test gap can be modeled by two series connected capacitors, resulting in a total capacitance as given by equation (2.2).

$$
C_{\text {tot }}=\frac{C_{a i r} C_{\text {sample }}}{C_{\text {air }}+C_{\text {sample }}}
$$

where, $C_{\text {sample }}$ and $C_{\text {air }}$ are the complex capacitances of the sample material and the air inside the electrode gap. From equation (2.2), the sample capacitance can be calculated if the air gap and the total capacitances are known:

$$
C_{\text {sample }}=\frac{C_{a i r} C_{t o t}}{C_{a i r}-C_{t o t}}
$$

The air gap capacitance can be obtained from the air reference measurement, described in the next section, by correcting for the changed distance. Similar approaches were mentioned in $[6,7]$. In this paper we are however additionally using air as reference to calibrate the measurement instrument.

\subsection{AIR REFERENCE METHOD}

In the air reference method, one measures the complex capacitance of a test cell under the same frequencies and the same test conditions twice, with and without presence of the tested material. The complex permittivity of the tested material is then calculated from the ratio of the two measured complex capacitances, the volume of the tested material and the dimension of the test cell. A similar method was utilized in [13].

In order to achieve a significant difference between the two measured capacitances and to have a reliable reference, the first measurement is made on the capacitance $\left(C_{l}\right)$ of two parallel electrodes (area $a_{l}$ and gap distance $d_{l}$ ) with only air in between. This measurement can then be looked on as an air reference calibration. According to the literature, the relative permittivity of air under normal condition is

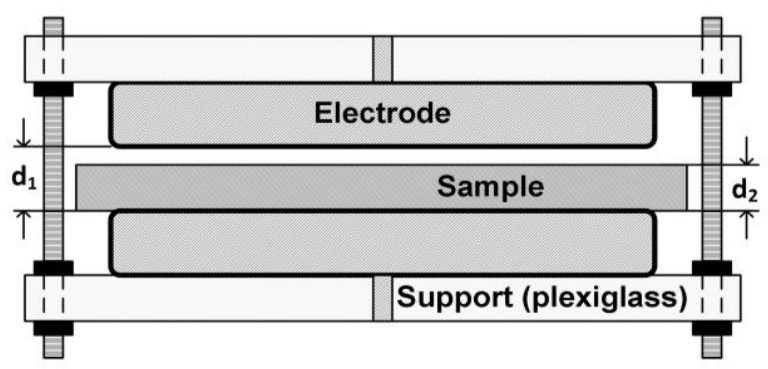

Figure 4. Sketch of the contact-free electrode arrangement, $d_{1}$ is the distance between two parallel electrodes and $d_{2}$ is the thickness of tested material.

$1.00058986 \pm 0.00000050[14]$ and the loss factor is assumed to be small but dependent on humidity. However, for the present accuracy requirements, the relative permittivity of air can be set to unity with no losses. If the required accuracy is higher, the measurements can be made in vacuum. In practice, it is not necessary to perform this air calibration measurement for every sample characterization, but it should preferably be measured when the testing conditions change, such as the current shunt impedance shows drift, the distance between the electrodes is modified, the testing cell is moved or the temperature varies.

The second capacitance $\left(C_{2}\right)$ is the capacitance of the identical test cell as in the first calibration measurement but with a material sample (with area $a_{2}, a_{2}>a_{1}$; thickness $d_{2}$, $d_{2} \leq d_{1}$ ) in the electrode gap. The capacitance $C_{2}$ is given by $C_{t o t}$ of equation (2.2). Together with the first measured air capacitance $C_{l}$, which gives the air gap capacitance in equation (2.3) as $d_{1} /\left(d_{1}-d_{2}\right) C_{1}$, the complex permittivity and dissipation factor of the material can be calculated through equation (2.4)

$$
\left\{\begin{array}{l}
\varepsilon_{r}=\varepsilon_{r}^{\prime}-j \varepsilon_{r}^{\prime \prime}=d_{r} \frac{C_{\text {Sample }}}{C_{1}}=\frac{d_{r} K}{\left(d_{r}-1\right) K+1} \\
\tan \delta=\frac{\varepsilon_{r}^{\prime \prime}}{\varepsilon_{r}^{\prime}}
\end{array}\right.
$$

where $K=\frac{C_{2}}{C_{1}}=\frac{Z_{1}}{Z_{2}}, \quad d_{r}=\frac{d_{2}}{d_{1}}$

Here, $C_{l} / d_{r}$ is the partial capacitance of the air volume that is replaced by the sample. The relative permittivity is thus calculated in relation to the permittivity of the gas actually filling the electrode chamber and this is a disadvantage of all reference methods.

Though it is not possible to characterize the current shunt impedance $\left(Z_{s h}\right)$ in fine detail, the material permittivity can still be calculated with precision as the impedance ratio $(K)$ from the two measurements is used in equation (2.4). From equation (2.1), it follows that the result does not depend on the shunt impedance if the same current shunt is used in both measurements. This does not mean that the shunt characteristics are unimportant, however. The shunt impedance is decisive for the signal to noise ratio and thus determines the resolution of loss factor determination. The thermal drift that causes the shunt impedance change is one main error source in our air reference measurement. 


\subsection{ERROR ANALYSIS}

The permittivity and loss factor resolutions will be degraded by the existence of the air gap. This accuracy loss can be quantified by analysis of the error sources of contact-free electrode arrangement with respect to contact measurement.

From equation (2.4), two error sources can be identified. One is the distance measurement error which will be present in $d_{r}$, another error source comes from the electrical measurement noise and drift which will be present in the complex $K$ measurement. Here, one should note that the drift in amplitude and angle of $K$ are not always the same therefore they should be considered separately. The use of an air reference eliminates voltage and current sensing errors which must be considered in standard approaches.

Surface roughness and thickness variation will influence the resolution of the dielectric characterization and this is the main error source for contact measurements. Thus, it should always be evaluated in the error analysis. Such an evaluation is simplified by the observation that surface roughness and thickness variation can be viewed as an additional source of distance measurement error. Thus, the appropriate thickness average, $d$, for the capacitance of a test object with a varying thickness $d(x, y)$ over its surface, $x$ and $y$ being surface coordinates, is defined by the total capacitance

$$
C=\frac{\varepsilon_{r} \varepsilon_{0} A}{d}=\iint \frac{\varepsilon_{r} \varepsilon_{0}}{d(x, y)} d x d y
$$

which gives

$$
\frac{1}{d}=\frac{1}{A} \iint \frac{1}{d(x, y)} d x d y
$$

where the integration is over the electrode surface area $A$.

With these two independent error sources, the resulting error in permittivity is calculated as

$$
\Delta\left(\varepsilon_{r}\right)=\left|\frac{\partial \varepsilon_{r}}{\partial d_{r}}\right| \Delta\left(d_{r}\right)+\left|\frac{\partial \varepsilon_{r}}{\partial K}\right| \Delta(K)=\delta_{d} \Delta\left(d_{r}\right)+\delta_{K} \Delta(K)
$$

Where $\Delta\left(d_{r}\right)$ and $\Delta(K)$ are distance measurement and electrical measurement error estimates respectively, $\delta_{d}$ and $\delta_{K}$ are

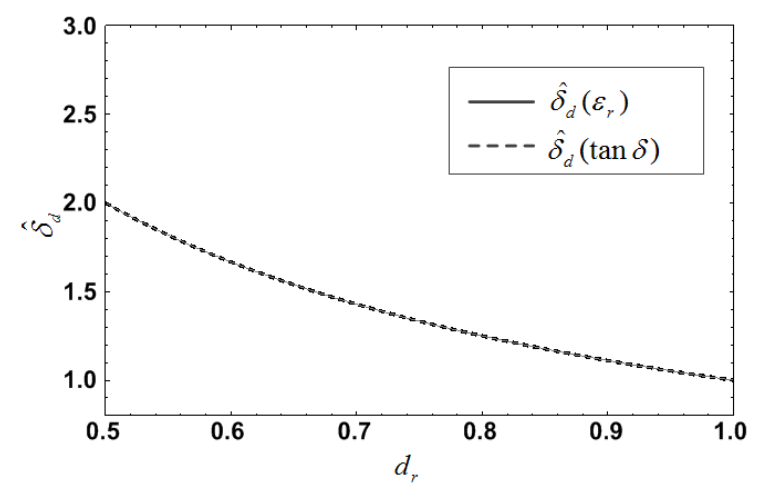

Figure 5. Normalised distance sensitivity parameter, $\hat{\delta}_{d}$ as a function of the gap fill factor, $d_{r}$. The results for $\varepsilon_{\mathrm{r}}$ and $\tan \delta$ are the same irrespective of the permittivity and the loss factor. sensitivity parameters. Distance measurement errors influence however also contact measurement, therefore a proper evaluation of the error increase due to contact free measurements should only consider the error increase due to the air gap $\hat{\Delta}\left(\varepsilon_{r}\right)$, which is found by forcing the sensitivity parameters to unity when $d_{r}=1$ :

$$
\begin{aligned}
& \hat{\Delta}\left(\varepsilon_{r}\right)=\hat{\delta}_{d} \Delta\left(d_{r}\right)+\hat{\delta}_{K} \Delta(K) \\
& \hat{\delta}=\delta\left(d_{r}\right) / \delta\left(d_{r}=1\right)
\end{aligned}
$$

thus defining the normalized sensitivity parameters $\hat{\delta}$.

An expression for the error increase due to distance measurement error, $\delta_{d}$, as defined by equation (2.5), can be found from equation (2.4) by first differentiating by $d_{r}$ and then substituting $K$ with $\varepsilon_{r}$ :

$$
\delta_{d}=\frac{\left(\varepsilon_{r}-1\right) \varepsilon_{r}}{d_{r}}
$$

Here it is clear that there is an error increase also at $d_{r}=1$, due to the permittivity. The noise sensitivity parameter, $\delta_{K}$, is found from equation (2.4) in the same way.

$$
\delta_{K}=\left|\frac{\partial \varepsilon_{r}}{\partial K}\right|=\frac{\left(\left(1-d_{r}\right) \varepsilon_{r}+d_{r}\right)^{2}}{d_{r}}
$$

This is unity at $d_{r}=1$, reflecting that the noise influence is the same for all permittivities in contact measurements.

A similar analysis can be performed for the error increase in the loss factor, $\tan \delta$. This is slightly complicated by the separation of the real and imaginary parts of $K$ but otherwise very similar, for space reasons the expressions are not presented here. For small loss factors, the result is approximately the same as equation (2.9).

To exemplify the error increase magnitudes, the normalized sensitivity parameters for some selected permittivity are presented in Figures 5 and 6.

As illustrated in Figure 5, the increase in $\varepsilon_{r}$ and $\tan \delta$ errors due to distance ratio measurement error $\Delta\left(d_{r}\right)$ relative to the

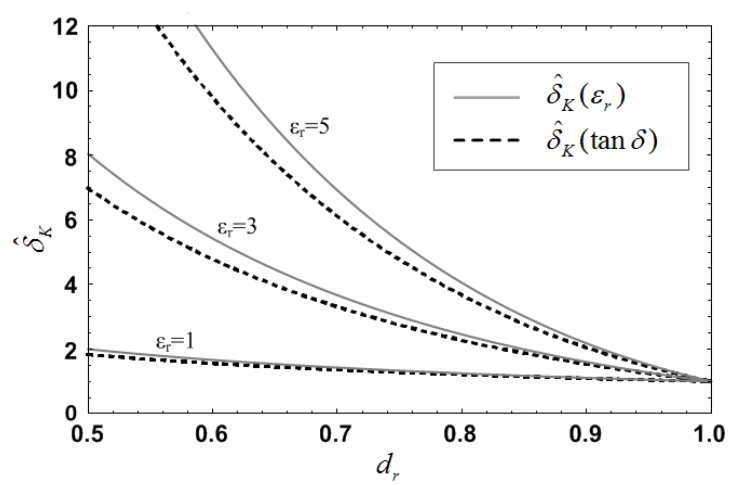

Figure 6. Normalised noise sensitivity parameter, $\hat{\delta}_{K}$, as a function of the gap fill factor, $d_{r}$. The solid lines are valid for $\varepsilon_{\mathrm{r}}$ and the dashed lines for $\tan \delta$, with $\varepsilon_{\mathrm{r}}=5,3,1$ from top of figure respectively. In the figure, a loss factor of $10 \%$ has been used to make the difference visible. Already at $1 \%$ loss the curves for $\varepsilon_{\mathrm{r}}$ and $\tan \delta$ are almost identical. 
contact measurement increase with decreasing distance ratio $d_{r}$ and they are independent on $\varepsilon_{r}$.

The second error source, electrical measurement noise and drift, causes error increases as illustrated in Figure 6. In this case, the increases in both permittivity and loss are $\varepsilon_{r}$ dependent. As a worst case, an order of magnitude in precision may be lost for a half filled electrode gap with materials of interest.

Even though the contact-free air reference method will amplify the errors from both distance and electrical measurements, the elimination of the sensing circuit characterization is a major error-reducing factor. In some cases, especially for small losses, systematic errors in the sensing characterisation dominate the loss factor error, as will be exemplified below. This is because it is very difficult to characterise electrical components with a precision of $0.01 \%$ or better.

A formal error analysis, as presented above, provides the possibility to analyse error propagation consequences in general, as illustrated in Figures 5 and 6 above. This is of great value, especially when designing a measurement. For practical measurement error estimates on actual data sets, however, a simpler and more direct method is to process the original data with a set of values for the critical parameters that cover the estimated uncertainty range. Such an approach further serves as a confirmation of the formal error analysis.

In these considerations, we have neglected an aspect that will cause some systematic errors in the permittivity magnitude. When material samples of different permittivity are present between the electrodes, the effective electrode area will namely change due to distortion of the electric field at the electrode edges. To some extent, this effect can be controlled by geometric factors such as the electrode edge radius, electrode area to distance ratio and introduction of guard rings. For precision measurements of the permittivity, the edge field distortion needs nevertheless careful consideration and is therefore the subject of our ongoing studies. In this paper, we are however mainly focusing on the loss factor, which is not affected by this effect to first order.

\section{MEASUREMENT DESIGN}

A contact-free electrode arrangement using the air reference method can be utilized by any dielectric response instrument with enough high resolution. As the air reference method eliminates detailed modeling of the analog circuit, this calibration principle can also be beneficial with contact electrode arrangements, where it may improve accuracy. Thus, two different instruments, in the following denoted as AWIS and IDAX, were used to verify this method with both contact and contact-free electrode arrangements. As a comparison, the direct method is also used in IDAX measurements with a contact electrode arrangement.

\subsection{EXPERIMENTAL SETUP}

The AWIS (Arbitrary Waveform Impedance Spectroscopy) instrument uses a versatile voltage-current measurement technique for dielectric material studies $[15,16]$. It can not only measure a dielectric response under voltages with a specified frequency, but is also capable of determining an entire dielectric spectrum from one measurement, provided the test voltage is rich enough in harmonics. The IDAX instrument (IDAX-300) [17] is a commercial insulation diagnostic analyzer. It applies a sinusoidal voltage with the desired frequency over the sample and by accurately measuring the voltage and resulting current, the total impedance can be obtained. At this level of abstraction, both AWIS and IDAX operate similarly. The main practical difference between them is that IDAX measures responding currents with several electrometers (operational amplifiers), feedback capacitor and balancing capacitor [18]. AWIS, on the other hand, employs a current shunt to obtain responding currents [16]. Both have their advantage and disadvantage. Operational amplifier has negligible impedance but its frequency response spectrum is limited, whereas, a capacitive shunt provides a much broader frequency response but require a much higher impedance.

The used AWIS setup consists of an arbitrary waveform generator (HP 33120A) which provides the applied voltage $V_{0}$, a shunt $\left(Z_{s h}\right)$ which converts the current response into a measureable voltage $V_{l}$, and a multiplexing DAQ card (NI USB-6251) which is used to measure $V_{0}$ and $V_{l}$. To ensure low source impedance for the current measurement, which is required with multiplexing DAQ cards, the $V_{l}$ signal is passed through a buffer amplifier.

The current shunt $\left(Z_{s h}\right)$ is built by a capacitor in parallel with a resistor. The advantage of a capacitive shunt is that the impedance of the shunt is matched with the impedance of the test object.

The used IDAX instrument is an insulation diagnostic system mainly intended for analysis of power apparatuses but it may also be used for material characterization. Sinusoidal voltages up to $200 \mathrm{~V}_{\text {peak }}$ in the frequency range from $0.1 \mathrm{mHz}$ to $10 \mathrm{kHz}$ can be applied to the test object. According to the manual [17], the accuracy of the IDAX-300 instrument for capacitance measurement is $0.5 \%+1 \mathrm{pF}$ and the accuracy of the loss factor from $1 \mathrm{mHz}$ to $100 \mathrm{~Hz}$ is $1 \%+0.0003$, from $100 \mathrm{~Hz}$ up to $1 \mathrm{kHz}$ is $2 \%+0.0005$. The loss factor accuracy from $1 \mathrm{kHz}$ up to $10 \mathrm{kHz}$ is not specified.

In each IDAX measurement, one pre-measurement is performed to select among several current sensitivities for different frequency bands in order to obtain optimal response amplitude. For the AWIS measurements, there is no need to change shunt because of its high sensitivity and the capacitive shunt arrangement.

The used test cell is made up of two flat bare stainless steel electrodes $(r=47 \mathrm{~mm}$ ) which are supported by two plexiglass plates and three screws, as shown in Figure 4. The distance between the two electrodes can be adjusted by modifying the position of nuts on the screws. The test cell is fixed in a shielded metal box to minimize the external influence.

\subsection{MEASUREMENT PROCEDURE}

The relative permittivity and loss factor as function of frequency are calculated from the AWIS and the IDAX measured results using both contact and contact-free electrode arrangements. 
In the AWIS measurements, the air reference method has to be used for both contact and contact-free electrode arrangements, as the current shunt is not characterized. Therefore, the impedance ratio $(K)$ is obtained from the applied and response voltages $\left(V_{O}\right.$ and $\left.V_{l}\right)$ by eq. 2.1, and thereafter is used in eq. 2.4 to calculate the complex permittivity of the material.

In the IDAX measurements, the ratio of the two measured complex capacitances $\left(C_{1}\right.$ and $\left.C_{2}\right)$ is directly used in eq. 2.4 to calculate the complex permittivity of the material by the air reference method with both contact and contact-free electrode arrangements.

The traditional, direct, dielectric response measurement with contact electrode arrangement can also be performed with the IDAX instrument. The relative permittivity and loss factor are then directly calculated from the measured sample capacitance $\left(C_{2}\right)$ and the dimensions of the test cell.

\section{RESULTS AND ERROR ESTIMATION}

An absolute verification of the improved measurement accuracy by the air reference method is difficult to obtain. This would require comparison to an independent measurement method of high precision. For the loss factor, a calorimetric method could be considered, for example. Such methods are however at least as intricate as the proposed method and they need verification. As no such method is available to us, we have to resort to comparisons using different FDS instruments and materials to, at least, obtain an indication of measurement precision.

In contrast, the contact-free electrode arrangement can easily be verified by a comparison with a contact measurement. In such a comparison, increased losses in the contact measurement are expected at higher frequencies due to the possible existences of series resistances and these should increase linearly with frequency.

Two dielectric materials, polycarbonate and aluminum tri hydroxide (ATH) filled ethylene-propylene-dienemonomer (EPDM) rubber are used to exemplify the air reference method in application of the contact-free electrode arrangement. These materials have very different properties, EPDM rubber is much softer than polycarbonate. For comparisons, samples were also measured by the direct method with contact electrode arrangement. Before each measurement, samples were carefully washed by isopropanol and left to dry under room conditions for one hour.

The errors in sample measurements are then estimated based on the noise and drift observed in the air reference measurement as well as the estimated error of distance measurement.

\subsection{ERROR ESTIMATION}

Error estimation in practical measurement is performed based on the original measured data with a set of values for the critical parameters that cover the estimated uncertainty range. The two identified error sources which define the error range are estimated as discussed below.

The distance measurement error as well as surface roughness
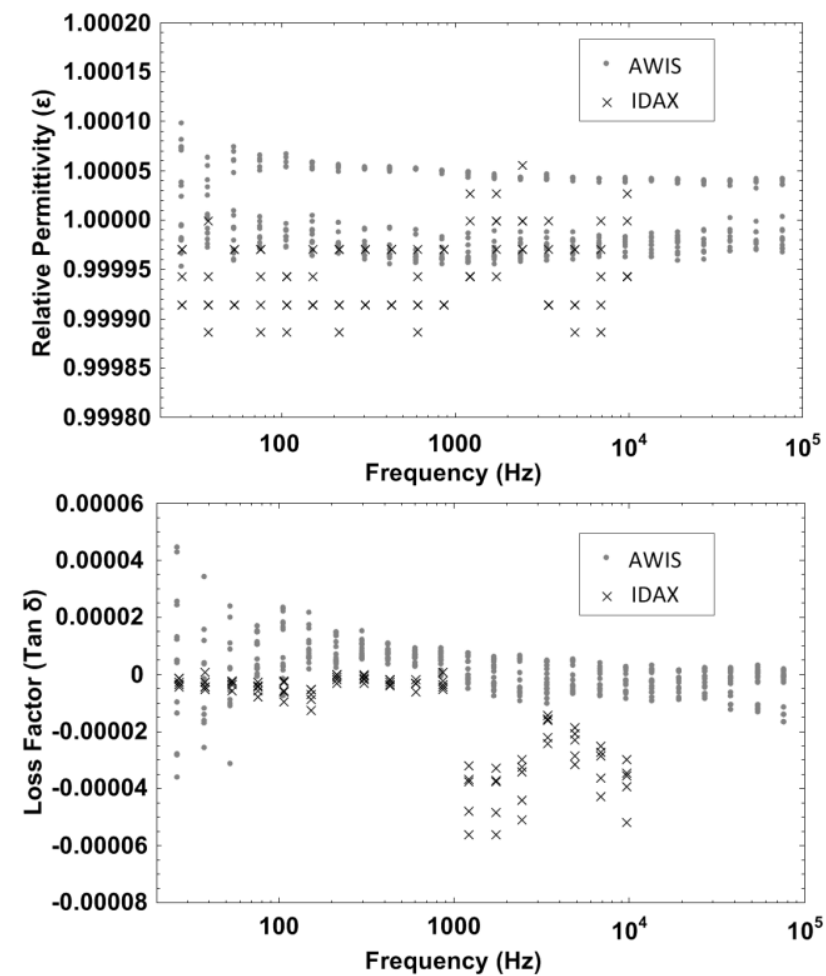

Figure 7. The measured relative permittivity and loss factor of air by air reference method with 3 different gap distances. It is indicated that the base error due to electrical measurement noise and drift in permittivity measurements are $\pm 0.01 \%$ for AWIS and IDAX; in $\tan \delta$ measurements these are $\pm 0.005 \%$ for both.

can be estimated by the accuracy of the measurement instrument, the distance of the measurement gap and the thickness of sample. In our measurements, a micrometer with accuracy of $0.005 \mathrm{~mm}$ is used.

The base error due to electrical measurement noise and drift can be estimated by measuring one identical electrode air gap twice and performing the air reference calculation. By repeating this measurement procedure several times, the deviation of air permittivity and loss factors can be taken as a measure of the error. Figure 7 shows the results of air permittivity and loss factor measured by air reference measurements with three different gap distances, each air gap was swept 5 times. The results indicate that the base error due to electrical measurement noise and drift is about $\pm 0.01 \%$ in permittivity measurement for both AWIS and IDAX, in $\tan \delta$ measurement $\pm 0.005 \%$ for both instruments. As indicated in the manual, IDAX is less well calibrated above $1 \mathrm{kHz}$ which is visible in the loss factor plot of Figure 7. Then, the real error due to electrical measurement noise and drift can be calculated using the estimated sample permittivity and calculated noise sensitivity as described in section 2.5 or by performing the analysis several times using values of $d_{r}$ and $K$ within the uncertainty range. It has been verified that both methods give the same result.

\subsection{POLYCARBONATE}

The specimen was a $0.75 \mathrm{~mm}$ thick polycarbonate sample, Bayer's GP 0099. It is a comparatively stiff material and the surface of the sample was polished. According to the material datasheet [19], the loss factor of the sample is 0.0005 at $1 \mathrm{kHz}$, 
which is roughly the IDAX claimed accuracy level.

The relative permittivity and loss factor results of the sample, which were measured with the air reference method, are shown in Figure 8. As a comparison, results directly obtained by IDAX are also shown. The estimated error range for each measurement is also indicated in the figure, where, for the direct IDAX measurement, the sensing accuracy as given by the manual dominates. A good agreement is found between the results of relative permittivity obtained by means of the two different instruments when applying the air reference method, while slightly different results may be noticed for the results obtained directly by IDAX. This difference is within the sensing error range. The error estimation indicates that errors in permittivity measurements with contact electrode arrangement are roughly the same for both direct and air reference methods. A good agreement is also found in the results of loss factor measured by the air reference method. However, a significant difference is exhibited between the results of air reference method and the directly measured loss factor by IDAX. From the error estimation, it is seen that the air reference method has much less error than the direct method. This can be taken as an indication of accuracy improvement provided by the air reference method due to elimination of the sensing characteristics which dominates the direct measurement.

We have noticed in our experiments that the sensitivities of IDAX and AWIS are different. In order to measure losses in
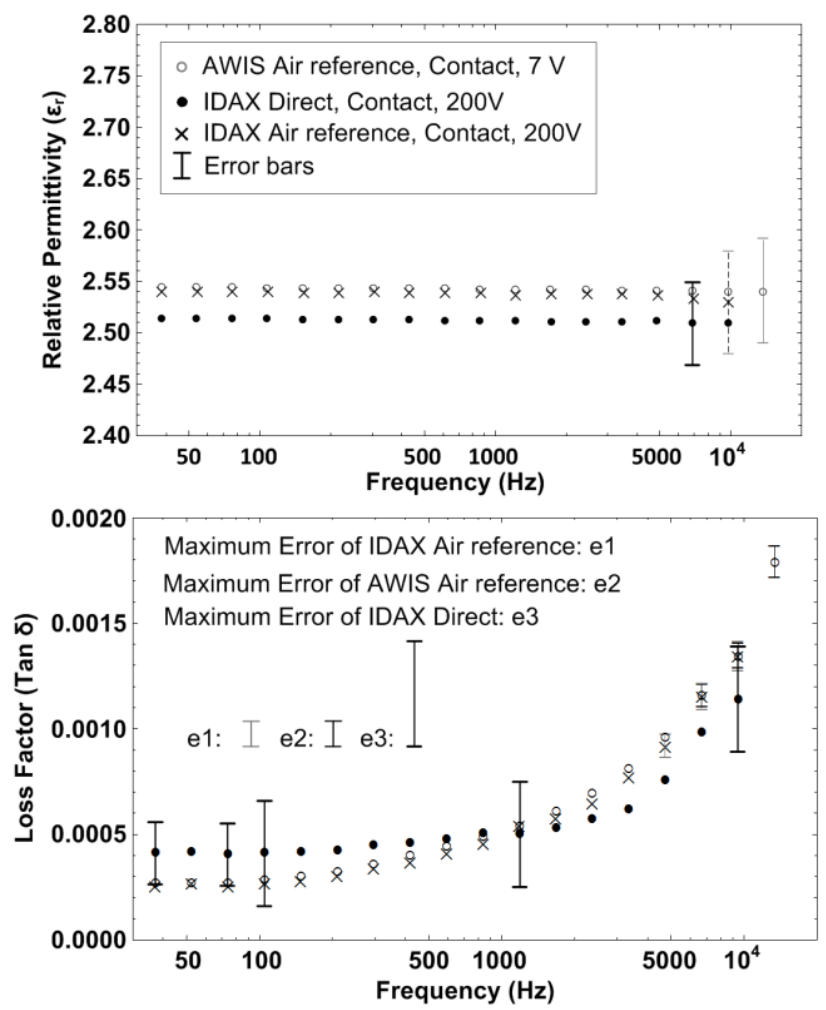

Figure 8. The relative permittivity and loss factor of a $0.75 \mathrm{~mm}$ thick polycarbonate sample measured by AWIS with the air reference method and measured by IDAX with both the air reference method and the traditional method. In the measurements, the contact electrode arrangement was used. In order to improve the readability, only few data points are marked with error bars as they are in a same uncertainty range for each frequency. the $0.1 \%$ range, AWIS needs a lower level of excitation voltage than IDAX, $7 \mathrm{~V}$ and $200 \mathrm{~V}$ were respectively needed for reaching this accuracy, which indicates the advantages of the greater optimization possibilities of AWIS.

The polycarbonate sample was also measured with the contact-free electrode arrangement with an electrode distance of $1.5 \mathrm{~mm}$. In Figure 9, the relative permittivity and loss factor results are compared with the results obtained from contact measurement.

A small difference is visible in the permittivity results, most probably related to effects of the effective electrode area. This difference is the subject of our further investigations. The error estimation shows that errors in permittivity measurements with contact-free electrode arrangements are larger than the error range of contact measurement as only half of the air gap was filled with the sample. It is interesting to note that the loss factor values of the contact-free electrode arrangement are lower than the results obtained with the contact electrode arrangement, especially at higher frequencies. This effect is expected to arise from the existence of a series resistance at the sample surface, as illustrated in Figure 2, and is thus taken as an indication, but not a proof, of increased precision by contact-free measurements. The results also indicate that the error range for contact-free measurement is larger than the error range of contact air reference measurement but smaller than the difference due to the sample surface contact.
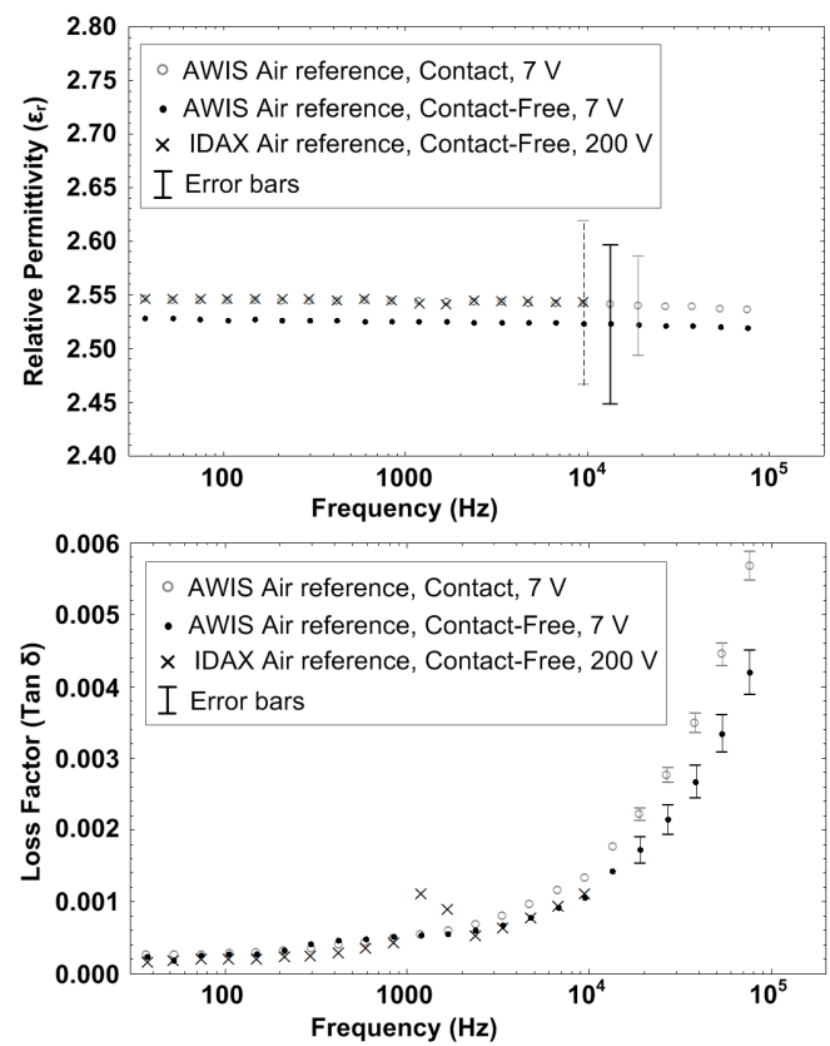

Figure 9. Relative permittivity and loss factor of a $0.75 \mathrm{~mm}$ thick polycarbonate sample measured by the AWIS with contact and contactfree electrode arrangement and measured by the IDAX with contactfree electrode arrangement, similar to Figure 8 . In the measurements, the air reference method was applied. 
In the loss factor results, two frequency points in the IDAX results deviate strongly from the overall trend. The reason for this deviation can be due to the automatic change of current sensitivity in IDAX. To use the air referencing method, it must be ensured that the same sensing characteristics are used in both measurements at each frequency. If not, the error is only limited by the sensing accuracy, which may be much larger than the error from noise and drift.

\subsection{EPDM WITH ATH FILLER}

To further evaluate if the air reference method and the contact-free electrode arrangement are valid for characterizing of dielectric materials with different properties, an ATH filled EPDM rubber was also tested. This sample had a thickness of $1.93 \mathrm{~mm}$ and the surface roughness $\mathrm{R}_{\mathrm{a}}<5 \mu \mathrm{m}, \mathrm{R}_{\mathrm{z}}<10 \mu \mathrm{m}$ according to Dektak profilometer measurement.

The sample was measured with the air reference method in both contact and contact-free electrode arrangements with an electrode distance of $2.57 \mathrm{~mm}$.

In Figure 10, loss factors of the two AWIS results are compared. As a reference, the IDAX measured result is also shown. Agreement is found in the results with the contact electrode arrangement measured by AWIS and IDAX respectively. The results with the contact-free electrode arrangement exhibit again a lower loss factor as compared to the results with contact electrode arrangement, probably due to the influence of surface resistances. Thus, these results show similar behavior as those obtained on polycarbonate sample.

\section{CONCLUSION}

In this paper, we have discussed two techniques intended to improve dielectric material characterization, namely air reference method and contact-free electrode arrangement.

The air reference method can improve instrument accuracy by performing a calibration with a known specimen, air, under the same conditions as the material is tested. Thus, the detailed voltage and current sensing properties are eliminated from the results. This requires that the instrument have the same sensitivity in the reference and the sample measurements, which is not guaranteed for commercial instruments using automatic sensitivity selection for improving direct

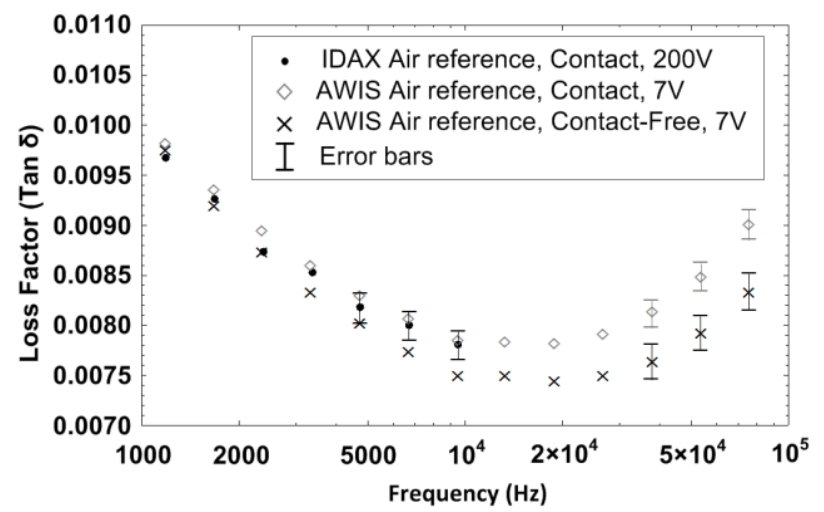

Figure 10. Loss factor of a $1.93 \mathrm{~mm}$ thick EPDM with ATH filler sample measured by AWIS and IDAX with different electrode arrangements, similar to figure 8 . In all the measurements, the air reference method was applied. measurement accuracy. Our results indicate a substantial improvement in accuracy as compared to direct measurements. In particular, this method may improve loss factor sensitivity into ranges required for characterization of modern insulation materials.

Contact-free measurements are proposed as a solution to the electrode contact problem, which often requires intricate and time-consuming procedures to be under control. To apply a contact-free measurement, the air reference method is required as the dielectric properties of the sample are found from the ratio of two capacitance measurements. There is inevitably some loss of sensitivity by contact-free measurements as an air gap contribution need to be eliminated from the result. Thus, these measurements demand a higher intrinsic sensitivity than direct measurements whereas they may significantly reduce sample preparation time and improve reproducibility. The air referencing method eliminates however one important source of error, the sensor calibration error. In cases where this error dominates, an improved accuracy may be obtained despite the loss of accuracy due to the air gap.

Studies of further improvements of the contact-free method by accounting for the different electric field distribution at the electrode edge as well as surrounding geometric influences are under way.

\section{ACKNOWLEDGMENT}

This work has been carried out within ELEKTRA project 36085 financed jointly by the Swedish Energy Agency, Elforsk and ABB.

\section{REFERENCES}

[1] W. S. Zaengl, "Dielectric spectroscopy in time and frequency domain for $\mathrm{HV}$ power equipment. I. Theoretical considerations," IEEE Electr. Insul. Mag., Vol. 19, Iss. 5, pp. 5-19, 2003.

[2] W. S. Zaengl, "Applications of dielectric spectroscopy in time and frequency domain for HV power equipment," IEEE Electr. Insul. Mag., Vol. 19, Iss. 6, pp. 9-22, 2003.

[3] R. J. Mammone and M. Binder, "Novel methods for preparing thin, high permittivity polymer dielectrics for capacitor applications," in 34th Int'l Symp. Power Sources Cherry Hill, NJ , USA, pp. 395-398, 1990. T. A. Prevost, "Dielectric Properties of Natural Esters and their Influence on Transformer Insulation System Design and Performance," in IEEE PES Conf. and Exhi. Transmission and Distribution, Calgary, AB, pp. 30-34, 2006.

M. K. W. Stranges, R. Omranipour, and N. Stranges, "A Comparison of Dielectric Characteristics and Voltage Endurance Life for Two High Voltage Coil Designs," in IEEE Int'l Symp. Electrical Insulation (ISEI), Vancouver, BC, pp. 223-226, 2008.

[6] E.T.Hoch, "Electrode effects in the measurement of power factor and dielectric constant of sheet insulating materials," Bell System Tech. Jour., Vol. 5, Iss. 4, pp. 555-572c, Oct. 1926. 
[7] Recommended methods for the determination of the permittivity and dielectric dissipation factor of electrical insulating materials at power, audio frequency including metre wavelengths, IEC 250, 1969.

[8] A. Castro-Couceiro, M. Sánchez-Andújar, B. RivasMurias, J. Mira, J. Rivas, and M. A. SeñarísRodríguez, "Dielectric response in the chargeordered Ca2-xPrxMnO4 phases," Solid State Sciences, Vol. 7, Iss. 8, pp. 905-911, 2005.

[9] H. Provencher, B. Noirhomme, and E. David, "Effect of aging on the dielectric properties of insulation paper of power transformers," in IEEE Conf. Electrical Insulation (EIC), pp. 473-477, 2009.

[10] P. Tharning and U. Gafvert, "High voltage dielectric frequency response measurements on polyethylene samples during water tree ageing," in IEEE 5th Int'1 Conf. Conduction and Breakdown in Solid Dielectrics (ICSD), pp. 671-675, 1995.

[11] J. Mijovic, J. Kenny, A. Maffezzoli, A. Trivisano, F. Bellucci, and L. Nicolais, "The principles of dielectric measurements for in situ monitoring of composite processing," Composites Science and Technology, Vol. 49, Iss. 3, pp. 277-290, 1993.

[12] W. P. Baker and F. B. Waddington, "Dielectric measurements on thin films by means of electrodes coated with irradiated silicone rubber," J. Sci. Instrum., Vol. 36, pp. 309-312, 1959.

[13] X. Hairu, Z. Yewen, and Z. Feihu, "Study on measuring method of dielectric spectroscopy for polymer dielectrics," in IEEE 9th Int'l Conf. Properties and Applications of Dielectric Materials (ICPADM) pp. 922-925, 2009.

[14] L. G. Hector and H. L. Schultz, "The Dielectric Constant of Air at Radiofrequencies," Physics, Vol. 7, Iss. 4, pp. 133-136, 1936.

[15] B. Sonerud, T. Bengtsson, J. Blennow, and S. M. Gubanski, "Dielectric response measurements utilizing semi-square voltage waveforms," IEEE Trans. Dielectr. Electr. Insul., Vol. 15, Iss. 4, pp. 920926, 2008.

[16] X. Xu, T. Bengtsson, J. Blennow, and S. M. Gubanski, "Harmonic Limited Test Waveforms for Fast AWIS Dielectric Studies," presented at the 22nd Nordic Insul. Sympos. (NORD-IS), Tampere, Finland, pp. 199-202, 2011.

[17] IDAX User's Manual: Megger AB, 2009.

[18] P. Werelius, P. Tharning, R. Eriksson, B. Holmgren, and U. Gafvert, "Dielectric spectroscopy for diagnosis of water tree deterioration in XLPE cables," IEEE Trans. Dielectr. Electr. Insul., Vol. 8, Iss. 1, pp. 27-42, 2001.

[19] Production data sheet Makrolon GP Soild ploycarbonate sheet, October 2004.

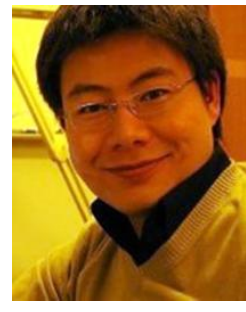

Xiangdong Xu (S'12) was born in 1984. He received the B.Sc. (Electrical engineering) and B.Eng. (Software engineering) from the Southwest Jiaotong University Chengdu, China, in 2006 and 2007, respectively. In 2009, he received the M.Sc. from Chalmers University of Technology, Gothenburg, Sweden. Then, $\mathrm{He}$ was working with grid asset management in University of Southern Denmark until 2010. He is currently pursuing the Ph.D. degree at Chalmers University of Technology. His research interests include dielectric response measurement techniques, dielectric material characterization, partial discharge and grid asset management.

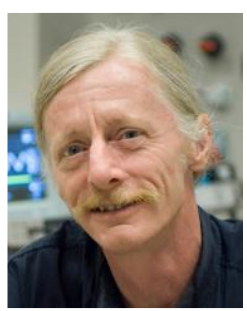

Tord Bengtsson (M'06) received the Ph.D. degree in mathematical physics from Lund Institute of Technology in 1984 and continued working with theoretical nuclear physics until 1991, when he joined ABB Corporate Research in Västerås, Sweden. Since then, he has been actively engaged in developing diagnostic methods for high voltage apparatuses and systems. Some particular interests are acoustic methods, partial discharges and signal processing. In 2006, he was appointed as a part time professor in High Voltage Engineering at Chalmers University of Technology in recognition of his efforts to develop research on insulation properties under voltages with fast rise times.

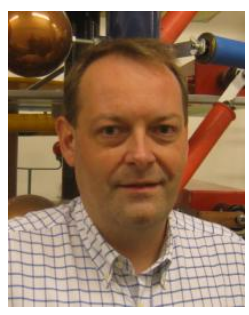

Jörgen Blennow (S'94-M'01) was born in 1966. He graduated with the M.Sc. in electrical engineering from Chalmers University of Technology, Gothenburg, Sweden in 1993. He obtained the Ph.D. degree in 2000 in high voltage engineering after which he became an Assistant Professor in 2006 and Senior Lecturer in 2006. From 2012 he is an Associate Professor in High Voltage Engineering at the same university. His main research activities have been towards different aspects of insulation exposed to high frequency high voltage, air-solid insulation systems, partial discharges, cable insulation and transformer diagnostics based on dielectric spectroscopy.

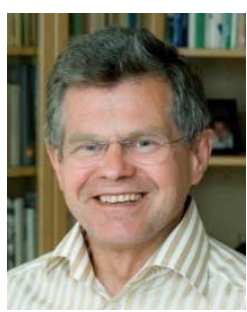

Stanislaw M. Gubanski (M'89-SM'90-F'01) received the M.Sc. (high voltage engineering) and Ph.D. degrees (material science) from the Technical University of Wroclaw, Poland, in 1973 and 1976, respectively. He was a Research Fellow at the University College of North Wales Bangor, U.K from 1976 to 1977, and a senior lecturer at the Technical University of Wroclaw, Wroclaw, Poland, from 1977 to 1988. Afterwards he was associate professor (19891996) at the Royal Institute of Technology, Stockholm, Sweden. Currently, he is professor in High Voltage Engineering at the Department of Materials and Manufacturing Technology, Chalmers University of Technology. He is a Senior Associate Editor of the IEEE Transactions on Dielectrics and Electrical Insulation. 